\title{
Rectangular Waveguide Filters with Meandered Topology
}

\author{
Fernando Teberio, Member, IEEE, Jon M. Percaz, Ivan Arregui, Petronilo Martin-Iglesias, \\ Txema Lopetegi, Miguel A. G. Laso, and Israel Arnedo, Senior Member, IEEE
}

\begin{abstract}
In this paper, a new topology for rectangular waveguide band-pass and low-pass filters is presented. A simple, accurate and robust design technique for these novel meandered waveguide filters is provided. The proposed filters employ a concatenation of $\pm 90^{\circ}$ E-plane mitered bends $\left( \pm 90^{\circ} \mathrm{EMBs}\right)$ with different heights and lengths whose dimensions are consecutively and independently calculated. Each $\pm 90^{\circ} \mathrm{EMB}$ satisfies a local target reflection coefficient along the device so that they can be calculated separately. The novel structures allow to drastically reduce the total length of the filters and to embed bends if desired, or even to provide routing capabilities. Furthermore, the new meandered topology allows the introduction of transmission zeros above the passband of the low-pass filter, which can be controlled by the free parameters of the $\pm 90^{\circ} \mathrm{EMBs}$. A band-pass and a low-pass filter with meandered topology have been designed following the proposed novel technique. Measurements of the manufactured prototypes are also included to validate the novel topology and design technique, achieving excellent agreement with the simulated results.
\end{abstract}

Index Terms - Band-pass filter, low-pass filter, impedancematching, routing, stepped-impedance, waveguide filter.

\section{INTRODUCTION}

$\mathrm{R}$ ECTANGULAR waveguide filters are key components in multiple practical applications, with special presence in the fields of radar and communications [1]-[5]. The choice of the final implementation technology depends on the specific application and its needs in terms of insertion loss, weight or power handling. Rectangular waveguide technology is widely used in satellite communication payloads when low insertion loss and high-power handling capability are required, despite its mass and volume. Therefore, the reduction of both troublesome characteristics in any application, but specifically in the satellite ones, is considered highly advantageous. The

This paper is an expanded version from the IEEE MTT-S International Microwave Workshop Series on Advanced Materials and Processes (IMWSAMP 2017), Pavia, Italy, 20-22 September 2017.

This work was supported by ESA's Networking/Partnering Initiative (NPI) under Contract 4000114859/15/NL/HK, Gobierno de Navarra under Project 0011-1365-2017-000130, and MINECO (Spain) under Projects TEC201451902-C2-2-R and TEC2017-85529-C3-2-R

Fernando Teberio, Jon M. Percaz, Ivan Arregui, Txema Lopetegi, Miguel A. G. Laso, and Israel Arnedo are with the Electrical and Electronic Engineering Department, Public University of Navarre, Pamplona E-31006, Spain (e-mail: fernando.teberio@unavarra.es).

Petronilo Martin-Iglesias is with the European Space Agency ESAESTEC, Noordwijk, The Netherlands. trends of a greater number of filters that must be accommodated in the payload, particularly in multi-beam satellites [6], and the increase of the the carrier frequencies are already a reality. Similarly, the industry is demanding reductions in manufacturing costs pursuing to eliminate tuning screws and guarantee successful first-shot fabrications. Finally, in such applications, waveguide bends can often be found after the filters [6], [7], misusing the available volume/mass budget and increasing the insertion loss. Moreover, when two different structures are used (screwed by their flanges), an additional negative impact is produced on the Passive Intermodulation (PIM) parameter [8], [9]. Therefore, the development of new band-pass filters (BPFs) and low-pass filters (LPFs) is in the spotlight, as well as accurate and robust design techniques to allow designers to obtain structures that: a) do not require post-manufacturing tuning, b) are compact, and c) provide routing capabilities.

To the authors' knowledge, all efforts to fold waveguide filters have remained focused on BPFs. An early attempt to achieve curved filters was published in [10], where the design of curved inductively-coupled BPFs starts from the same inline prototype and the deviations caused by the curvature of the cavities are afterwards compensated by means of a fullwave optimization procedure. Then, the folded topology was intensively utilized to miniaturize BPFs in the lower $\mathrm{GHz}$ frequency range with the development of waveguide combline filters [11], [12] and ridge waveguide filters [13]. After that, based also on the folded structures, design procedures for $\mathrm{H}$ plane filters and diplexers were also proposed [14]-[17]. The common feature of all the above structures is that they all have a symmetry in the H-plane and, therefore, the main current lines are broken when they are manufactured following the standard procedure in two halves, which deteriorates the measured insertion loss and PIM [18]. Recently, folded BPFs with E-plane symmetry have been proposed [18]-[20]. In [18] and [19], a folded topology was presented as a flexible alternative to implement trisections. Then, the technique in [18] was extended to implement transmission zeros above and below the passband [20]. Although very high-performance structures can be obtained with the above methods, they might not be suitable for the design and manufacture of tuning-less inexpensive filters with stringent specifications for the emerging Q-V-bands (assuming standard milling as the fabrication technique) since they all use coupled resonant cavities to obtain the desired frequency response. 
Although compact high-power LPFs have been obtained in [21]-[23] an E-plane bend cannot be embedded in these types of filters. Hence, if an E-plane bend is required before or after the LPF, two different structures will be needed (with their corresponding flanges, screws, etc.). This issue will have a negative impact on the insertion loss, volume/weight, and PIM products due to the mandatory presence of extra junctions.

Stepped-impedance waveguide structures have been widely used for LPFs [24]-[34], and also for BPFs [24]-[28], [35], always featuring an in-line (straight) topology, and they still remain a very common solution for the implementation of waveguide LPFs. Fortunately, stepped-impedance BPFs are very good candidates as it was demonstrated in [35], where a straight BPF with reduced sensitivity to manufacturing tolerances was proposed. However, although the manufacturing yield of the structure was clearly enhanced, the price to pay was the total size of the final device.

In this paper, we extend [33] where the idea of meandering a stepped-impedance LPF was presented. Now, a detailed modular methodology with a clear physical insight, extended also to BPFs, allows us to design tuning-free compact filters, with the flexibility of embedding bends and providing routing capabilities in the filter structure. Furthermore, due to their modular design methodology, cascading $\pm 90^{\circ}$ E-plane mitered bends $\left( \pm 90^{\circ} \mathrm{EMBs}\right)$, the design procedure can be easily implemented in a software tool to directly obtain a bended filter with the required routing specifications. Last but not

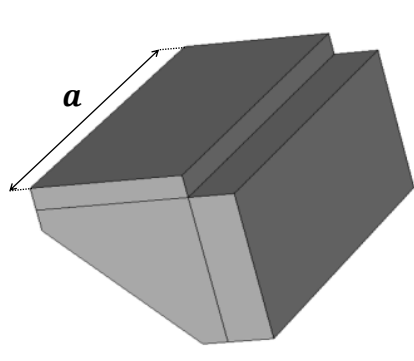

(a)

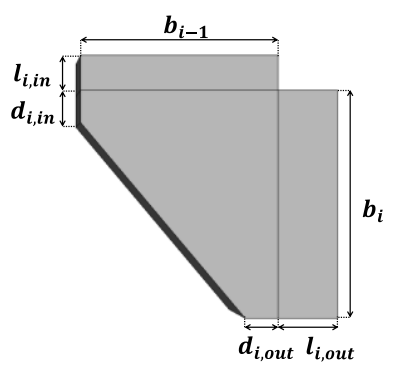

(b)
Fig. 1. Sketch view of the $i$ th $90^{\circ}$ EMB (a) 3-D view (b) side view.
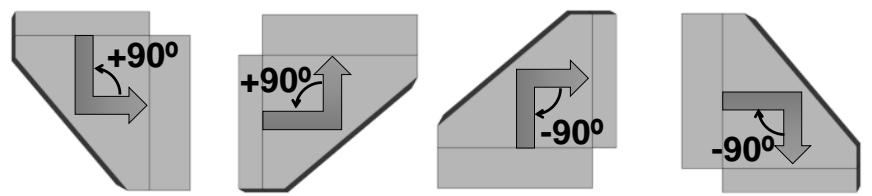

Fig. 2. Orientation of the bend and sign notation

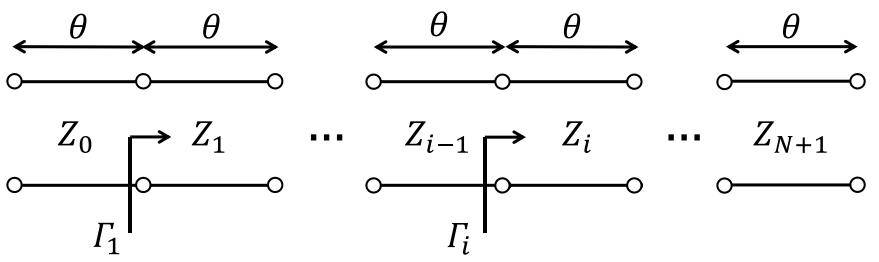

Fig. 3. Stepped-impedance prototype with commensurate transmission lines with electrical length $\theta$ and characteristic impedances $Z_{i}$. least, the novel meandered filters have E-plane symmetry, reducing the measured insertion loss and the PIM due to the fabrication assembly. The design method (Section II) has been demonstrated with two examples (Section III): a meandered BPF of order 13 (implemented with multiple topologies) with the same frequency specifications as in [35], and a meandered LPF of order 11, with transmission zeros, fulfilling the same specifications as in [33]. The LPF, and two BPF topologies, have been fabricated and measured, showing a very good agreement between simulated and measured results.

\section{DESIGN METHOD}

The novel meandered filters presented in this paper (of order $N$ for both the band-pass and low-pass responses) consist of the concatenation of $N+1 \pm 90^{\circ}$ E-plane mitered bends $\left( \pm 90^{\circ} \mathrm{EMBs}\right)$ with the same width, $a$, but different heights (input $b_{i-1}$ and output $b_{i}$ ), lengths (input $l_{i, \text { in }}$ and output $l_{i, \text { out }}$ ) and tilts of the chamfer (input $d_{i, \text { in }}$ and output $\left.d_{i, \text { out }}\right)$, where sub-index $i$ identifies the $i$ th-EMB, see Fig. 1 . The "+" or the "-" of the $\pm 90^{\circ} \mathrm{EMB}$ is in accordance with Fig. 2.

The design method begins by calculating the steppedimpedance prototype (distributed transmission line model), see Fig. 3, which consists of $N+2$ commensurate transmission lines with electrical lengths $\theta$ and characteristic impedances $Z_{i}$, (which produces $N+1$ impedance steps) for a chosen allpole frequency response of order $N$ such as Butterworth, Chebyshev, Zolotarev, Chained Function, etc. As it is well known, the commensurate lines have the same frequency behavior, since they are commensurate and homogeneous. In fact, all lines have ideally the same physical length $l$, and show the same wavelength $\lambda_{g}$ at any frequency. These properties allow us to define the frequency behavior of the prototype as a function of the electrical length of a line, $\theta$ :

$$
\theta=\beta l=\frac{2 \pi}{\lambda_{g}} \cdot l
$$

Each commensurate line has a different characteristic impedance $Z_{i}$ whose value can be calculated by applying the well-known Richards' transformation and the iterative extraction procedure fully detailed in [3]. Then, as it was explained in [32], a local reflection coefficient, $\Gamma_{i}$, is produced at each junction between two commensurate lines by the characteristic impedance mismatch, whose value is given by

$$
\Gamma_{i}=\frac{Z_{i}-Z_{i-1}}{Z_{i}+Z_{i-1}}
$$

The combination of all these local reflections placed $\theta \mathrm{rad}$ apart produces the aimed frequency response of the filter (see Fig. 4).

The synthesis technique proposed in this paper utilizes a modular design strategy. Thus, instead of paying attention to the final structure as a whole, each constituent $\pm 90^{\circ} \mathrm{EMB}$ (with its physical dimensions labeled in Fig. 1) will be designed separately and consecutively to resemble the electrical behavior of the transmission line model in terms of its local 
reflection coefficients, $\Gamma_{i}$, and electrical separations, $\theta$, to subsequently assemble the final structure. Thus, the $i$ th-EMB will implement a $\theta / 2$-transmission line subsection cascaded with another $\theta / 2$-transmission line subsection and the reflection coefficient, $\Gamma_{i}$, that appears between them, see Fig. 5. This $\Gamma_{i}$ depends mainly on the contrast between the $b_{i-1}$ and $b_{i}$ dimensions, taking into account that $\Gamma_{i}>0$ requires $b_{i-1}<b_{i}$ while $\Gamma_{i}<0$ needs $b_{i-1}>b_{i}$. On the other hand, the electrical lengths $\theta / 2$ implemented by the $i$ th-EMB will be dominated by the physical lengths $l_{i, \text { in }}$ and $l_{i, o u t}$. To ensure the full equivalence between the transmission line model and the $i$ th-EMB, the dimensions of the $i$ th-EMB are adjusted in such a way that its $S_{11}$-parameter satisfies $\left|S_{11, i}\right|=$ $\left|\Gamma_{i}\right|$ (see Fig. 6) and its $S$-parameter phases (see Fig. 6) verify (3) for a positive reflection coefficient $\left(\Gamma_{i}>0\right)$ :

$$
\phi_{i, S_{11}}=-\theta \quad \text { and } \quad \phi_{i, S_{21}}=-\theta
$$

and (4) for a negative reflection coefficient $\left(\Gamma_{i}<0\right)$ :

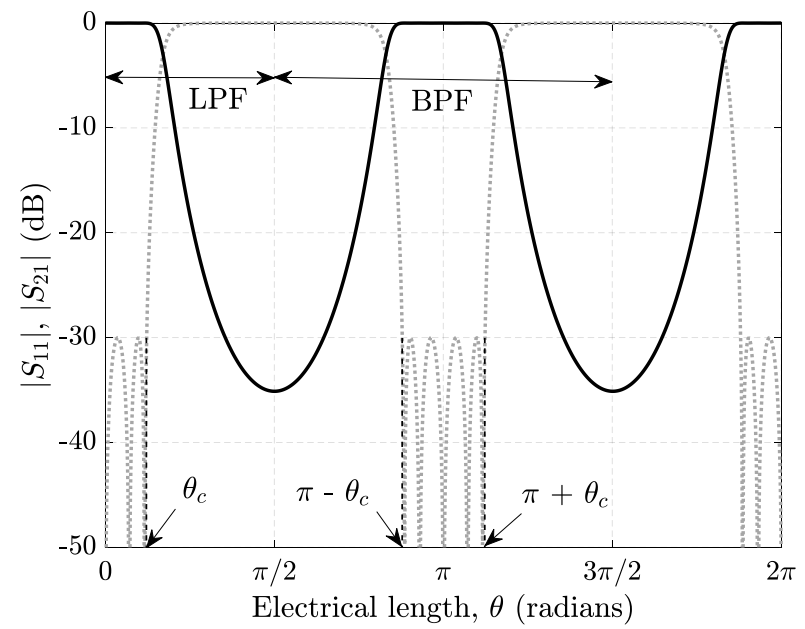

Fig. 4. 5-th order commensurate-line stepped-impedance prototype response for the LPF and BPF. $\left|S_{11}\right|$ (grey line) and $\left|S_{21}\right|$ (black line) parameters.

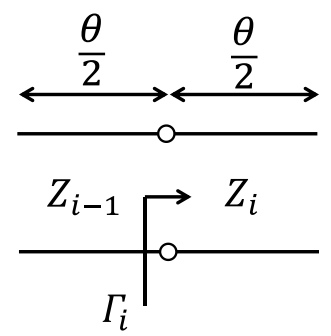

(a)

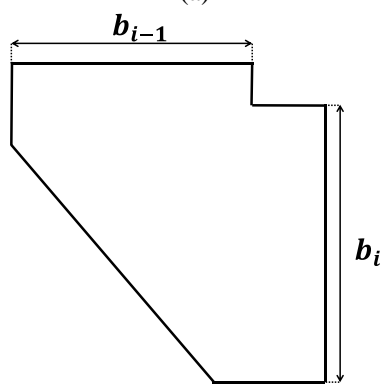

(b)

Fig. 5. Section of the stepped-impedance prototype (a) implemented by the $i$ th-EMB (b).

$$
\phi_{i, S_{11}}=-\theta+\pi \quad \text { And } \quad \phi_{i, S_{21}}=-\theta
$$

It is important to note that the dimensions that directly define the chamfer of the bend, $d_{i, \text { in }}$ and $d_{i, \text { out }}$, provide extra degrees of freedom in our design technique.

\section{A. Meandered BPF}

The novel meandered BPF (see Fig. 7) is designed utilizing the first inherent passband replica of the classical steppedimpedance low-pass prototype, centered at $\theta=\pi$ (see Fig. 4), [35]. The synthesis procedure begins by fixing the order of the filter, $N$, the required in-band return loss, and its lower and upper frequencies, $f_{1}$ and $f_{2}$, corresponding to $\theta=\pi-\theta_{c}$ and $\theta=\pi+\theta_{c}$, respectively, see Fig. 4, where $\theta_{c}$ is computed following (5).

$$
\theta_{c}=\pi \cdot \frac{\lambda_{g, f_{1}}-\lambda_{g, f_{2}}}{\lambda_{g, f_{1}}+\lambda_{g, f_{2}}}
$$

It is worth noting that the maximum rejection of the lower and upper stopbands takes place at $\theta=\pi / 2$ and $\theta=3 \pi / 2$, respectively. Then, we compute the transmission line model for the selected all-pole filtering function, extracting the commensurate lines or Unit Elements [3] (the collection of $N+2$ characteristic impedances $Z_{i}$ ) which will satisfy the required frequency specification, see Fig. 3. Now, the required local reflection coefficients, $\Gamma_{i}$, can be calculated using (2).

The procedure for obtaining the dimensions of each $\pm 90^{\circ} \mathrm{EMB}$ starts by fixing the width, $a$. Typically, this parameter is fixed as the standard port width. However, it can be reduced to enhance the fabrication yield of the filter as it was demonstrated in [35]. This is due to the fact that the electrical bandwidth of the filter will be wider for reduced widths. Thus, a higher value of $\theta_{c}$ will be needed, and the impedance ratios required for the same frequency

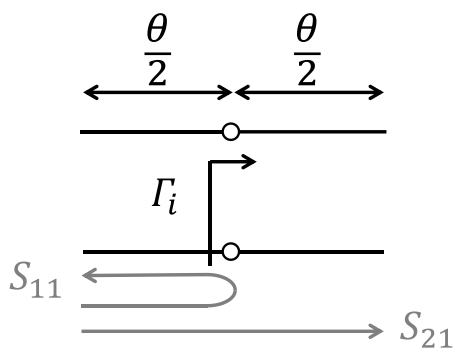

Fig. 6. Sketch of the $S_{11}$ and $S_{21}$ parameters corresponding to the section of the stepped-impedance prototype implemented by the $i$ th-EMB.

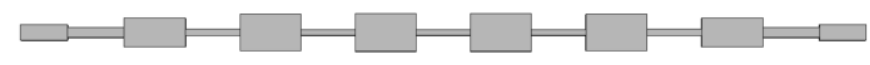

(a)

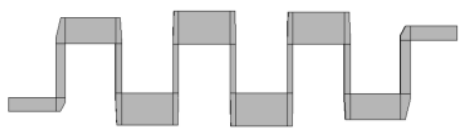

(b)

Fig. 7. Side-view (same scale) of (a) the classical straight waveguide BPF of order 13 [35] and (b) the novel meandered topology applied to the same filter. 
specifications will be reduced. Consequently, more relaxed fabrication tolerances are attained. This is particularly relevant for high frequency bands and for applications where narrow fractional bandwidths are required. However, it is important to note that this waveguide width reduction will produce a shifting towards the passband of the maximum rejection frequencies of the lower and upper stopbands.

The design method continues by mapping the $i$ th local reflection coefficient, $\Gamma_{i}$, calculated by (2), with the physical dimensions of the $i$ th-EMB, see Fig. 1 and Fig. 5. Initially, $b_{0}$ is fixed. Then $b_{1}$ is computed to satisfy that $\left|S_{11,1}\left(f_{\pi}\right)\right|$ provides the reflection value prescribed by $\left|\Gamma_{1}\right|$, where $\left|S_{11,1}\left(f_{\pi}\right)\right|$ is the magnitude of the $S_{11}$-parameter of the 1 st$90^{\circ} \mathrm{EMB}$ at $f_{\pi}$, which is calculated following (6).

$$
f_{\pi}=\frac{c}{2} \cdot \sqrt{\frac{1}{a^{2}}+\frac{\left(\lambda_{g, f_{1}}+\lambda_{g, f_{2}}\right)^{2}}{\lambda_{g, f_{1}}^{2} \cdot \lambda_{g, f_{2}}^{2}}}
$$

The previous magnitude value mainly depends on the heights $b_{0}$ and $b_{1}$ and, since $b_{0}$ has been already fixed, $b_{1}$ can be easily obtained by means of an EM software tool and taking into account that $\Gamma_{i}>0$ requires $b_{i-1}<b_{i}$ while $\Gamma_{i}<0$ needs $b_{i-1}>b_{i}$. There is also a slight, but appreciable, dependence on the shape of the chamfer through the $d_{1, \text { in }}$ and $d_{1, \text { out }}$. This extra degree of freedom is used to reduce the height excursion. Then, proceeding consecutively with the rest of the $\pm 90^{\circ} \mathrm{EMBs}$, once a certain $b_{i-1}$ is known, $b_{i}$ is calculated to ensure that:

$$
\left|S_{11, i}\left(f_{\pi}\right)\right|=\left|\Gamma_{i}\right|
$$

taking advantage also of the $d_{i, \text { in }}$ and $d_{i, \text { out }}$. When $i=N+1$, all $b_{i}, d_{i, \text { in }}$, and $d_{i, \text { out }}$ in the final structure have been calculated. It is important to note that the maximum height $b_{i}$ of the structure must be equal or smaller than the maximum allowed height, $b_{\max }$, calculated through (8) to avoid the resonance of the $\mathrm{TE}_{011}$ mode inside the structure up to a selected maximum frequency, $f_{\max }$. This is fulfilled by selecting appropriately the starting $b_{0}$.

$$
b_{\text {max }}=\frac{1 / 2}{\sqrt{\left(\frac{f_{\max }}{c}\right)^{2}-\frac{1}{\lambda_{g, f_{\pi}}^{2}}}}
$$

Next, we calculate the lengths $l_{i, \text { in }}$ and $l_{i, \text { out }}$ of the $i$ th-EMB (see Fig. 1). Following (3) and (4), depending on the type of step that we have in the transmission-line model, i.e., either positive or negative $\Gamma_{i}$, the lengths $l_{i, \text { in }}$ and $l_{i, \text { out }}$ will be adjusted to satisfy (9) or (10) respectively:

$$
\begin{array}{ccc}
\phi_{i, S_{11}}\left(f_{\pi}\right)=-\pi & \text { and } & \phi_{i, S_{21}}\left(f_{\pi}\right)=-\pi \\
\phi_{i, S_{11}}\left(f_{\pi}\right)=-\pi+\pi=0 & \text { and } & \phi_{i, S_{21}}\left(f_{\pi}\right)=-\pi
\end{array}
$$

being $\phi_{i, S_{11}}\left(f_{\pi}\right)$ and $\phi_{i, S_{21}}\left(f_{\pi}\right)$ the phase of the $S_{11^{-}}$and $S_{21^{-}}$ parameters, respectively, of the $i$ th-EMB at $f_{\pi}$. Since the $i$ th and the $(i+1)$ th-EMB share the height of their output and

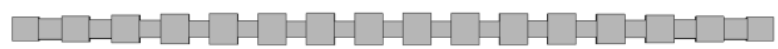

(a)

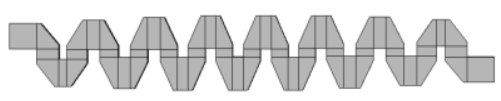

(b)

Fig. 8. Side-view (same scale) of (a) the classical straight waveguide LPF of order 29 [32] and (b) the novel meandered topology applied to the same filter.

input ports respectively, it is convenient to add the lengths $l_{i, \text { out }}$ and $l_{i+1, \text { in }}$ to obtain a single $l_{i}$ value following (11).

$$
l_{i}=l_{i, \text { out }}+l_{i+1, \text { in }} \quad \text { for } \quad i=1: N
$$

where the special cases for the global input and output lengths are calculated as $l_{0}=2 \cdot l_{1, \text { in }}$ and $l_{N+1}=2 \cdot l_{N+1, \text { out }}$.

Many possible topologies can be obtained depending on the sequence of $\pm 90^{\circ} \mathrm{EMBs}$ employed without the need of redesigning from scratch. If the selected sequence is $-90^{\circ} \mathrm{EMB}$, $+90^{\circ} \mathrm{EMB}, \quad+90^{\circ} \mathrm{EMB}, \quad-90^{\circ} \mathrm{EMB}, \quad-90^{\circ} \mathrm{EMB}, \quad+90^{\circ} \mathrm{EMB}$, $+90^{\circ} \mathrm{EMB}$, and so on, the most compact structure is obtained. This example is presented in Fig. $7 \mathrm{~b}$ in comparison with the in-line classical topology that is depicted in Fig. 7a.

\section{B. Meandered $L P F$}

The design method for the novel meandered LPF (see Fig. 8) begins by fixing the maximum frequency of the passband, $f_{c}$, the required in-band return loss, the frequency of maximum rejection, $f_{0}$, and the order, $N$, of the all-pole filtering function that will be employed. From the guided wavelength, $\lambda_{g}$, corresponding to $f_{c}$ and $f_{0}$, the value of $\theta_{c}$ will be obtained (see Fig. 4):

$$
\theta_{c}=\frac{\pi}{2} \cdot \frac{\lambda_{g, f_{0}}}{\lambda_{g, f_{c}}}
$$

Then the low-pass stepped-impedance prototype is computed by extracting the commensurate lines (Unit Elements) with their corresponding characteristic impedances $Z_{i}$ (see Fig. 3) which will satisfy the required frequency specifications. Next, the local reflection coefficients, $\Gamma_{i}$, are calculated using (2).

The design procedure continues by mapping each $\Gamma_{i}$ with the physical dimensions corresponding to the $i$ th-EMB (see Fig.1 and Fig. 5). Initially, $b_{0}$ is set according to the desired stopband performance and the minimum mechanical gap allowed in the filter (related to the power handling capability of the filter). Then, $b_{1}$ is calculated to satisfy $\left|S_{11,1}\left(f_{c}\right)\right|=$ $\left|\Gamma_{1}\right|$, where $\left|S_{11,1}\left(f_{c}\right)\right|$ is the magnitude of the $S_{11}$-parameter of the 1st-EMB at $f_{c}$, also taking into account that $b_{0}<b_{1}$ for $\Gamma_{1}>0$ and $b_{0}>b_{1}$ for $\Gamma_{1}<0$. After that, we proceed iteratively in such a way that once a certain $b_{i-1}$ is known, $b_{i}$ is computed to guarantee that:

$$
\left|S_{11, i}\left(f_{c}\right)\right|=\left|\Gamma_{i}\right|
$$


where $\left|S_{11, i}\left(f_{c}\right)\right|$ is the magnitude of the $S_{11}$-parameter of the $i$ th-EMB at $f_{c}$. When $i=N+1$, all $b_{i}$ have been calculated. Although the effect of the $b_{i}$ over the magnitude of the frequency response of the $\pm 90^{\circ} \mathrm{EMBs}$ is dominant, the effect of the $d_{i, \text { in }}$ and $d_{i, \text { out }}$ cannot be neglected. In the previous

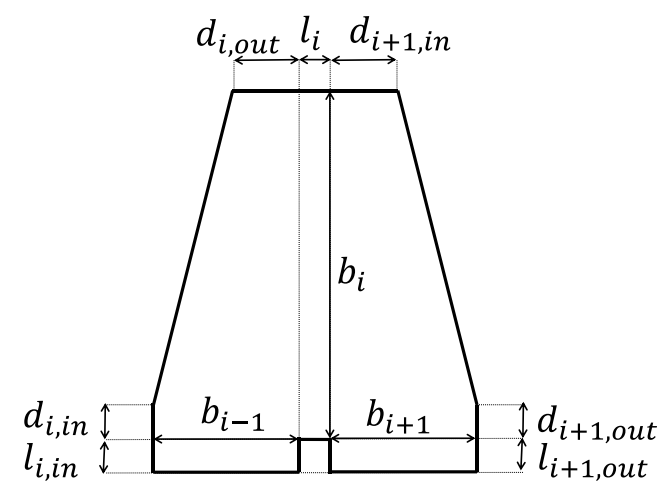

(a)

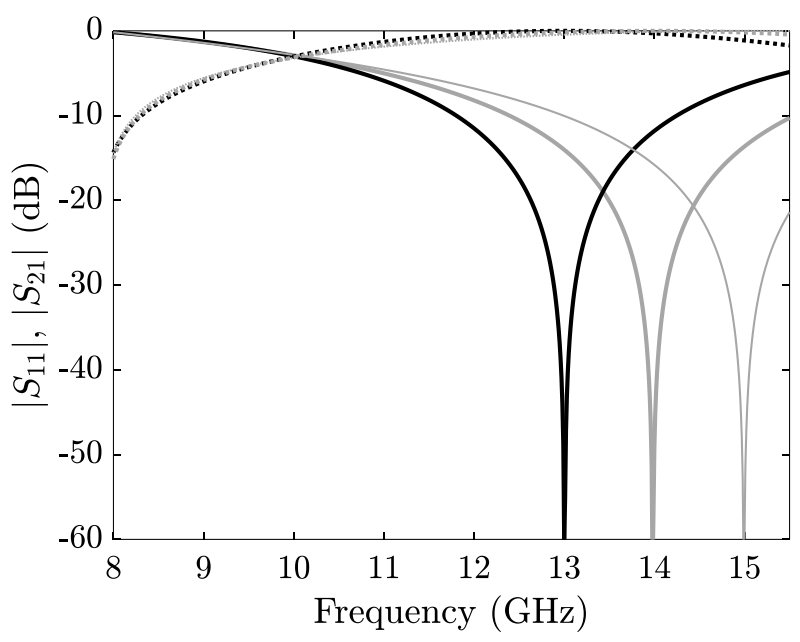

(b)

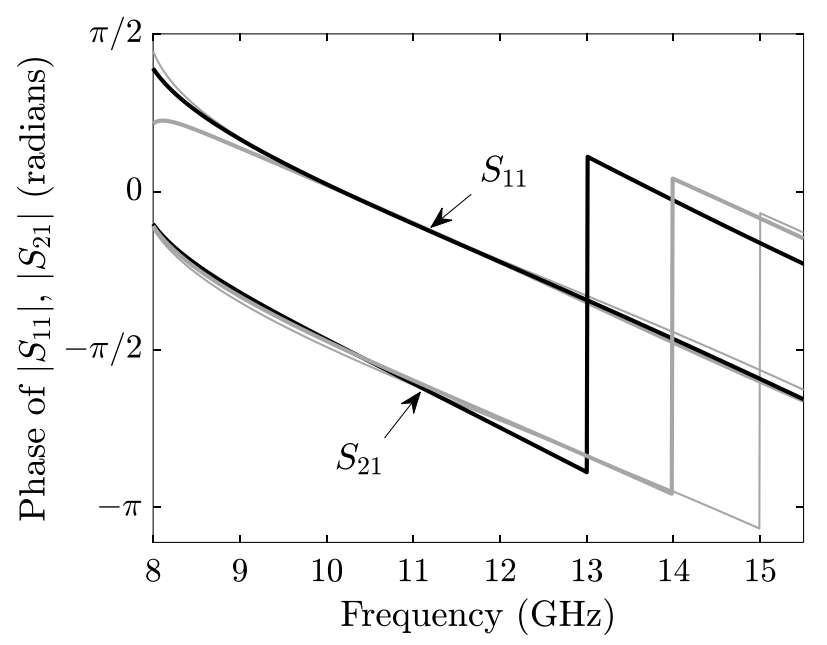

(c)

Fig. 9. Structure formed by two consecutive $-90^{\circ} \mathrm{EMBs}$, responsible of the introduction of a transmission zero (a). Frequency response of the structure for the three examples with dimensions detailed in Table I: Tx zero $13 \mathrm{GHz}$ (black line), Tx zero $14 \mathrm{GHz}$ (thick grey line), and Tx zero $15 \mathrm{GHz}$ (thin grey line); magnitude (b) and phase (c) procedure for the separated calculation of the $b_{i}$, two different strategies can be followed with respect to the $d_{i, \text { in }}$ and $d_{i, o u t}$. The first one is to a priori fix $d_{i, \text { in }}$ and $d_{i, \text { out }}$ in all the $\pm 90^{\circ} \mathrm{EMBs}$ to a constant value. The second one is to use, in each $\pm 90^{\circ} \mathrm{EMB}, d_{i, \text { in }}$ and $d_{i, \text { out }}$ along with $b_{i}$ (recalling that $b_{i-1}$ is fixed in the previous $\pm 90^{\circ} \mathrm{EMB}$ ) to obtain the required $\left|\Gamma_{i}\right|$. The advantage of this second option is that it allows the designer to control de required excursion for the $b_{i}$.

Finally, it just rests to compute the lengths $l_{i, \text { in }}$ and $l_{i, \text { out }}$ of the $\pm 90^{\circ} \mathrm{EMBs}$ (see Fig. 1). For doing so, the phase response of the $i$ th-EMB should be considered and, following (3) and (4), depending on the type of step that we have, i.e., either positive or negative $\Gamma_{i}$, the lengths $l_{i, \text { in }}$ and $l_{i, \text { out }}$ will be adjusted to satisfy (14) or (15) respectively:

$$
\begin{array}{ccc}
\phi_{i, S_{11}}\left(f_{c}\right)=-\theta_{c} & \text { and } & \phi_{i, S_{21}}\left(f_{c}\right)=-\theta_{c} \\
\phi_{i, S_{11}}\left(f_{c}\right)=-\theta_{c}+\pi & \text { and } & \phi_{i, S_{21}}\left(f_{c}\right)=-\theta_{c}
\end{array}
$$

being $\phi_{i, S_{11}}\left(f_{c}\right)$ and $\phi_{i, S_{21}}\left(f_{c}\right)$ the phase response of the $S_{11^{-}}$ and $S_{21}$-parameters, respectively, of the $i$ th-EMB at $f_{c}$. Finally, the $l_{i}$ are computed by means of (16), where the special cases for the global input and output lengths are calculated as $l_{0}=$ $2 \cdot l_{1, \text { in }}$ and $l_{N+1}=2 \cdot l_{N+1, \text { out }}$.

$$
l_{i}=l_{i, \text { out }}+l_{i+1, \text { in }} \quad \text { for } \quad i=1: N
$$

One remarkable property of the meandered LPFs proposed in this paper is the ability to introduce transmission zeros with this topology. It allows us to obtain very steep slopes between the pass- and the stop-band with a filter order reduction that will produce a substantial size reduction in comparison with the classical in-line structure [32] and with the meandered structure presented in [33], as it will be shown in the design examples Section. These transmission zeros are obtained by the interference between the fundamental $\mathrm{TE}_{10}$ operation mode and evanescent higher order modes which are excited at the bends. When we have two consecutive $-90^{\circ} \mathrm{EMBs}$ (or $+90^{\circ} \mathrm{EMBs}$ ), and the distance between the resulting input and output ports is small, see Fig. 9(a), the evanescent higher order modes excited can provide an alternative "path" for the signal to go from the input to the output port. When the electromagnetic fields that propagate through the main "path" (fundamental $\mathrm{TE}_{10}$ mode) interfere with the fields propagating through the alternative "path" (higher order modes) with the same strength but opposite phasing, then a cancellation occurs. This physical phenomenon produces a transmission zero at the cancellation frequency [15]. In order to obtain the transmission zero at a frequency close to the passband, the strength of the higher order mode fields that reach the output port must be high. To achieve it, the distance between the input and output ports must be small [15]. This can be accomplished by increasing $f_{0}$ (frequency of maximum rejection) to achieve a reduction in $\theta_{c}$, see (12), since this variable is directly connected to the length of each line and finally to the distance between the ports $l_{i}$. As a general rule, if the distance between the ports $l_{i}$ is decreased, the frequency of the transmission zero is also decreased, getting closer to the 
passband of the filter. Additionally, a fine tuning in the position of the zero can be achieved by changing the shape of the two bends through the adjustment of all their parameters, see Fig 9. To demonstrate the control that can be exerted over the position of the transmission zero, the two consecutive $-90^{\circ}$ EMBs structure of Fig. 9(a) has been designed for three examples, to provide the same frequency response (magnitude and phase) for a passband up to $11 \mathrm{GHz}$ approximately, while introducing a transmission zero at $13 \mathrm{GHz}, 14 \mathrm{GHz}$ and 15 $\mathrm{GHz}$, respectively. The physical parameters of the three examples are given in Table I and the frequency responses obtained are shown in Fig. 9. As it can be seen, the transmission zero can be shifted towards lower and higher frequencies around $2 \mathrm{GHz}$ (for these examples), while keeping the frequency response unaltered for the lower frequencies of the passband. Unfortunately, these transmission zeros cannot be achieved in the meandered BPFs, since the distance between the input and the output ports of the two consecutive $-90^{\circ} \mathrm{EMBs}$ structure of Fig. 9 is around $l_{i} \approx \lambda_{g, f_{\pi}} / 2$ for the BPFs, and cannot be reduced as in the novel meandered LPFs case.

Finally, it is important to note that the aim of the proposed meandered filters is to operate with the fundamental $\mathrm{TE}_{10}$ mode in the required passband and stopband. If the suppression of higher order modes is also desired in the stopband, a waveguide width modification (in the $x$-axis) following [34] could be also included.

The design methods presented in this paper are based on a simple modular procedure which can be easily introduced in a software tool. In fact, the $\pm 90^{\circ} \mathrm{EMBs}$ which compose the different structures are very simple, and the dimensions of each $\pm 90^{\circ} \mathrm{EMB}$ are obtained in a negligible computing time. At this point, it is important to note that the same kind of optimizations are necessary in the classical design process of in-line corrugated waveguide LPFs due to the effect of higherorder mode excitation at the discontinuities between the adjacent waveguides of different heights [30]. Moreover, the routing of the structure can be easily achieved with the proposed meandered filters, avoiding the use of subsequent bends, reducing the insertion loss, mass, and PIM. Additionally, the proposed techniques open the door to more complex routing structures with different angles.

\section{DESIGN EXAMPLES}

Two different filter specifications have been defined to demonstrate the performance of the proposed design techniques for the novel rectangular waveguide meandered filters: a Q-band BPF and a Ku-band LPF.

\section{A. Q-band Meandered BPF}

The novel meandered topology for rectangular waveguide BPFs has been proved by targeting the same state-of-the-art frequency specifications as in [35]. The meandered filter will have a passband between 37.5 and $42.5 \mathrm{GHz}$, with in-band return loss better than $20 \mathrm{~dB}$. The minimum attenuation required for the upper stopband $(47-50 \mathrm{GHz})$ will be $65 \mathrm{~dB}$. Additionally, a rejection level of $65 \mathrm{~dB}$ will be also required for the lower stopband (34-35 GHz).

In order to fulfil the previous frequency specifications, a meandered BPF will be designed using the novel technique explained in Section II.A. After including the usual design margin, a $13^{\text {th }}$ order Chebyshev function is chosen, with a return loss level of $25 \mathrm{~dB}$ on the passband defined between $f_{1}=37 \mathrm{GHz}$ and $f_{2}=43 \mathrm{GHz}$. The width of the rectangular waveguide is fixed to $a=4.65 \mathrm{~mm}$. The input port height has been set to $b_{0}=1.4 \mathrm{~mm}$, to avoid the coupling to the resonant $\mathrm{TE}_{011}$ mode inside the structure up to $f_{\max }=50 \mathrm{GHz}$. Finally, the free parameters that adjust the shape of the bends $d_{i, i n}$ and $d_{i, \text { out }}$ (see Fig. 1) have been fixed to $0.5 \mathrm{~mm}$ for all the $\pm 90^{\circ} \mathrm{EMBs}$, since they have no influence to obtain transmission zeros which are not possible in the BPF case.

Thus, using (5), the electrical length results in $\theta_{c}=$ $0.6938 \mathrm{rad}$ for our design, and applying the Unit Element extraction procedure the characteristic impedance list is obtained: $Z_{0}=Z_{14}=1, Z_{1}=Z_{13}=0.67, Z_{2}=Z_{12}=1.84, Z_{3}=$ $Z_{11}=0.41, Z_{4}=Z_{10}=2.35, Z_{5}=Z_{9}=0.37, Z_{6}=Z_{8}=2.46$, and $Z_{7}=0.36$. Next, using (2), the target reflection coefficients $\Gamma_{i}$ are calculated, and then the physical dimensions of each $\pm 90^{\circ} \mathrm{EMB}\left(b_{i}, l_{i, \text { in }}\right.$, and $\left.l_{i, \text { out }}\right)$ are computed to satisfy (7), (9), and (10). FEST3D is employed for the electromagnetic simulation and adjustment of each $\pm 90^{\circ} \mathrm{EMB}$. The final dimensions of the $\pm 90^{\circ} \mathrm{EMBs}$ are given in Table II (after applying (11)). To demonstrate the equivalence between the designed $\pm 90^{\circ} \mathrm{EMBs}$ and their corresponding sections of the stepped-impedance prototype (see Fig. 5), their frequency responses are compared in Fig. 10 for the 1st (initial) and the 7 th (central) $\pm 90^{\circ} \mathrm{EMBs}$. A remarkable wideband agreement is achieved between the frequency responses and the theoretical required values: $\left|\Gamma_{1}\right|=0.201$, $\left|\Gamma_{7}\right|=0.743$, see Table II, and $\phi_{1, S_{11}}\left(f_{\pi}\right)=\phi_{7, S_{11}}\left(f_{\pi}\right)=0$ and $\phi_{1, S_{21}}\left(f_{\pi}\right)=\phi_{7, S_{21}}\left(f_{\pi}\right)=-\pi$, see (10) and (4).

If the $\pm 90^{\circ} \mathrm{EMBs}$ are cascaded following a compact arrangement $(-++--++\cdots--+)$ in accordance with Fig. 7(b), the frequency response of the resulting meandered BPF (with no final optimization) is shown in Fig. 11. Its equivalent in-line BPF has been also designed following [35]. As it can be seen in Fig. 7, the total length of the structure has

TABLE I. DIMENSIONS FOR THE 3 EXAMPLES OF TWO CONSECUTIVE -90EMBS STRUCTURES WITH DIFFERENT TRANSMISSION ZEROS $(\mathrm{mm})$

\begin{tabular}{cccc}
\hline \hline $\begin{array}{c}\text { Design } \\
\text { Parameters }\end{array}$ & $\begin{array}{c}\text { Frequency of } \\
\text { Tx zero: } 13 \mathrm{GHz}\end{array}$ & $\begin{array}{c}\text { Frequency of } \\
\text { Tx zero: } 14 \mathrm{GHz}\end{array}$ & $\begin{array}{c}\text { Frequency of } \\
\text { Tx zero: } 15 \mathrm{GHz}\end{array}$ \\
\hline \hline$b_{i-1}$ & 4.229 & 4.229 & 4.229 \\
$b_{i}$ & 10.940 & 10.046 & 9.576 \\
$b_{i+1}$ & 5.253 & 4.229 & 3.936 \\
$l_{i, \text { in }}$ & 0.074 & 0.2 & 0.003 \\
$l_{i}$ & 0.150 & 0.818 & 1.458 \\
$l_{i+1, \text { out }}$ & 0.404 & 0.2 & 0.443 \\
$d_{i, \text { in }}$ & 1.380 & 1.547 & 1.466 \\
$d_{i, \text { out }}$ & 1.145 & 1.025 & 0.693 \\
$d_{i+1, \text { in }}$ & 1.814 & 1.947 & 2.246 \\
$d_{i+1, \text { out }}$ & 0.719 & 1.025 & 1.794 \\
\hline \hline
\end{tabular}


been significantly reduced. The length of the novel meandered BPF is only $37.6 \mathrm{~mm}$ vs. $81 \mathrm{~mm}$ for the in-line BPF (more than $50 \%$ length reduction). Finally, as it can be seen in Fig. 11, the simulated frequency response of both filters is in very good agreement. The novel meandered BPF has been fabricated in clam-shell configuration, in bare aluminum, by standard milling with a radius of the drill equal to $200 \mu \mathrm{m}$. A photograph of the prototype is shown in Fig. 11. In order to reach the standard WR22 ports for the measurements, two impedance transformers have been added to the input and output ports of the structure. The measurement results are also given in Fig. 11. A very good agreement between the simulated and the measured results is obtained.

One of the most remarkable features of the novel design method proposed in this paper is that, since it is a modular design procedure, once all the $\pm 90^{\circ} \mathrm{EMBs}$ are calculated, they can be connected as the designer desires. In order to demonstrate the high versatility and great flexibility of the meandered structures proposed, different topologies have been

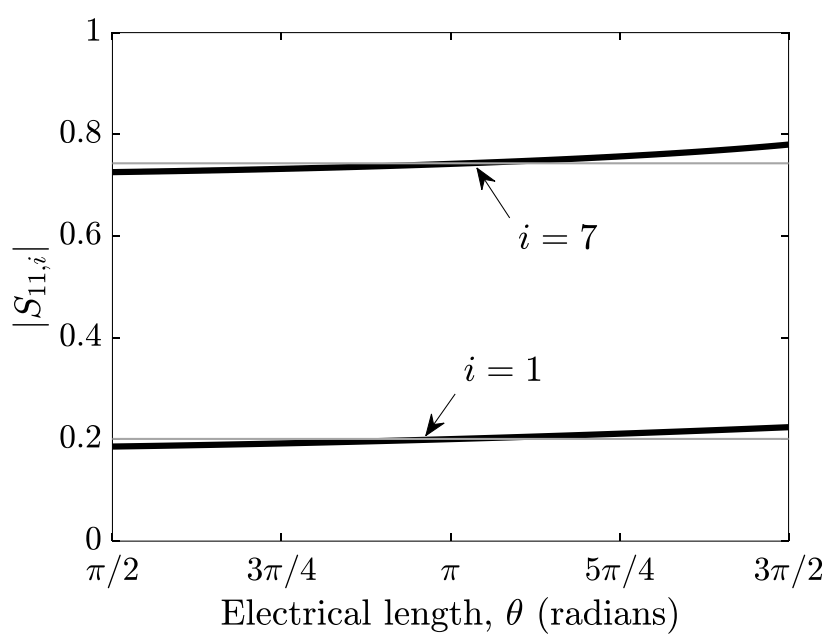

(a)

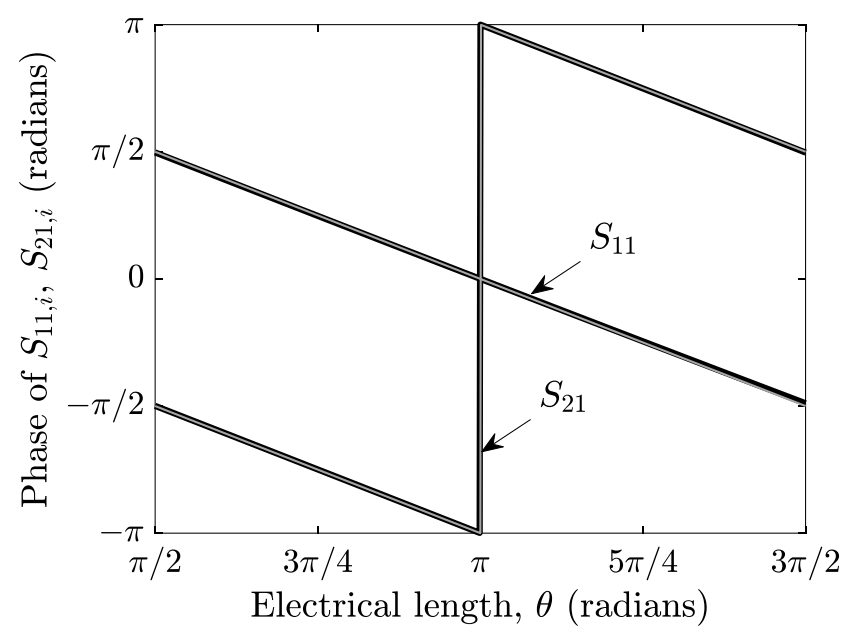

(b)

Fig. 10. (a) Magnitude and (b) Phase of the frequency response of the 1st and 7th EMBs designed for the Q-band meandered BPF (black thick line) vs. the frequency response of their corresponding sections of the stepped-impedance prototype (grey thin line). simulated, fabricated, and measured. Using the $\pm 90^{\circ} \mathrm{EMBs}$ designed for the example in Fig. 11 without any final optimization in their physical parameters (the physical dimensions employed are exactly those given in Table II), different ad-hoc layouts are proposed in Fig. 12 and Fig. 13. As it is shown in Fig. 12 and Fig. 13, the frequency response of both structures fulfils the specifications. Additionally, the meandered BPF of Fig. 13 has been fabricated and measured employing the same procedures detailed above. The measurement results are also given in Fig. 13. A good agreement between the simulations and the measurements is achieved, proving that filters with flexible routing capabilities can be obtained using the novel design technique and structures proposed in this work.

\section{B. Ku-band Meandered LPF}

In order to demonstrate the novel meandered topology for rectangular waveguide LPFs proposed in Section II.B, a compact LPF for Ku-band will be designed. The frequency

TABLE II

$\Gamma_{i}$ AND DIMENSIONS (mm) OF THE Q-BAND MEANDERED BPFS

\begin{tabular}{ccccccccc}
\hline \hline$\Gamma_{1}=-\Gamma_{14}$ & $\Gamma_{2}=-\Gamma_{13}$ & $\Gamma_{3}=-\Gamma_{12}$ & $\Gamma_{4}=-\Gamma_{11}$ & $\Gamma_{5}=-\Gamma_{10}$ & $\Gamma_{6}=-\Gamma_{9}$ & $\Gamma_{7}=-\Gamma_{8}$ \\
\hline-0.201 & 0.469 & -0.637 & 0.704 & -0.729 & 0.739 & -0.743 \\
\hline \hline$b_{0}=b_{14}$ & $b_{1}=b_{13}$ & $b_{2}=b_{12}$ & $b_{3}=b_{11}$ & $b_{4}=b_{10}$ & $b_{5}=b_{9}$ & $b_{6}=b_{8}$ & $b_{7}$ \\
\hline 1.400 & 0.971 & 2.547 & 0.602 & 3.191 & 0.546 & 3.322 & 0.540 \\
\hline \hline$l_{0}=l_{14}$ & $l_{1}=l_{13}$ & $l_{2}=l_{12}$ & $l_{3}=l_{11}$ & $l_{4}=l_{10}$ & $l_{5}=l_{9}$ & $l_{6}=l_{8}$ & $l_{7}$ \\
\hline 5.000 & 5.835 & 5.386 & 5.398 & 5.531 & 5.326 & 5.552 & 5.312 \\
\hline \hline
\end{tabular}

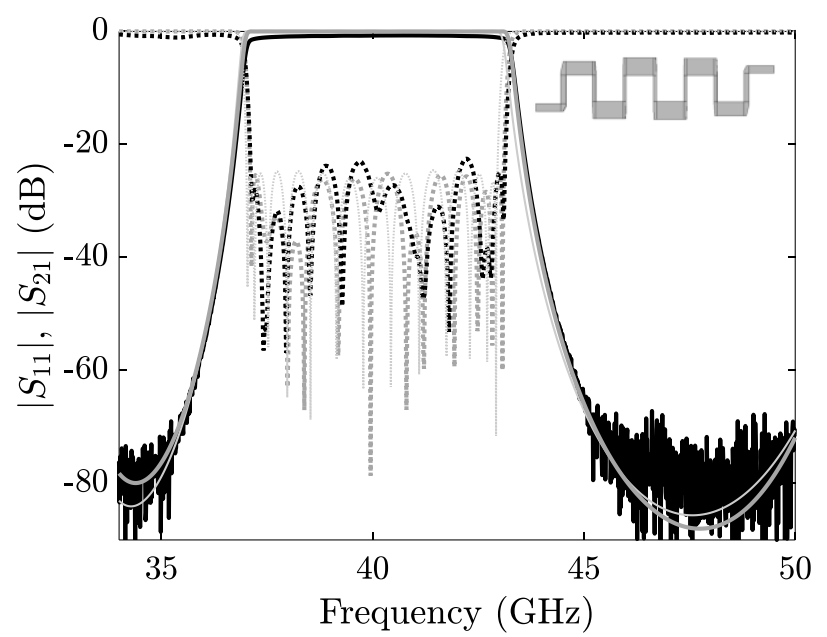

(a)
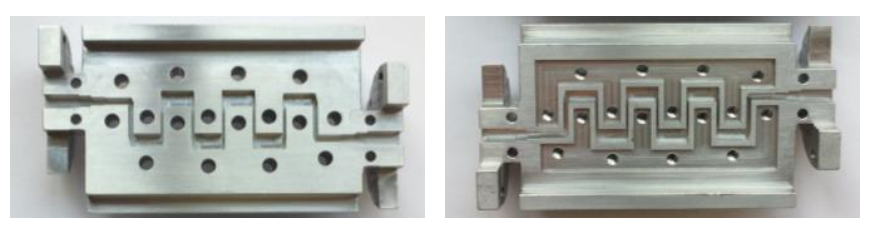

(b)

Fig. 11. (a) CST MWS simulated (grey thick line) and measured (black line) frequency response of the novel meandered BPF designed with the technique in Section II.A, and simulated frequency response of the in-line BPF (grey thin line). $\left|S_{I I}\right|$ in dotted line and $\left|S_{2 l}\right|$ in solid line. Inset: side-view of the simulated meandered BPF. (b) Photograph of the unassembled prototype. 
specifications will be the same as in [33]: passband defined between $10.7 \mathrm{GHz}$ and $11.7 \mathrm{GHz}$, with in-band return loss better than $20 \mathrm{~dB}$, and stopband defined between $13.75 \mathrm{GHz}$ and $14.5 \mathrm{GHz}$, with attenuation level higher than $60 \mathrm{~dB}$.

To fulfil the previous frequency specifications, a $11^{\text {th }}$ order Chebyshev function will be employed. The maximum frequency of the passband, $f_{c}$, and the frequency of maximum rejection, $f_{0}$, are set equal to $f_{c}=11.85 \mathrm{GHz}$ and $f_{0}=17 \mathrm{GHz}$.

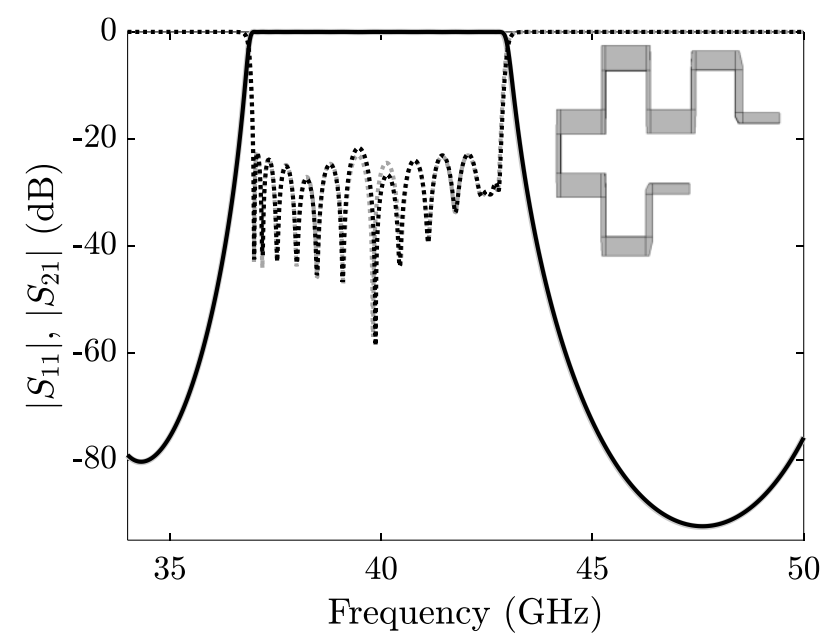

Fig. 12. Simulated frequency response of a different topology of meandered BPF designed with the technique in Section II.A. FEST3D simulation in grey line and CST MWS in black line. $\left|S_{11}\right|$ in dotted line, $\left|S_{2 l}\right|$ in solid line. Inset: side-view of the simulated BPF.

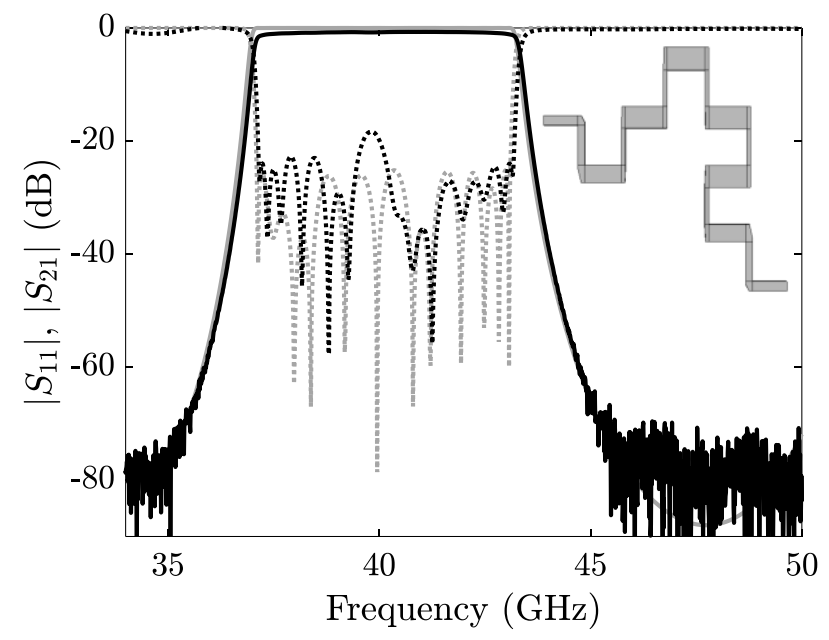

(a)

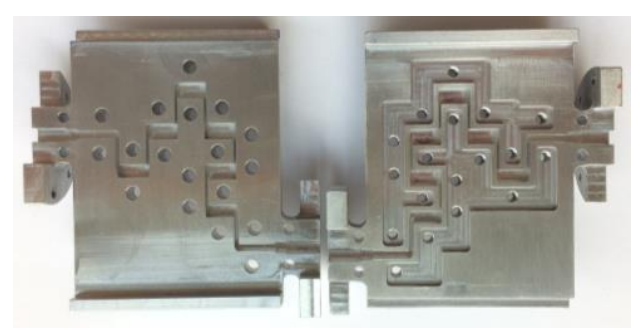

(b)

Fig. 13. (a) CST MWS simulated (grey thick line) and measured (black line) frequency response of the novel meandered BPF designed with the technique in Section II.A. $\left|S_{I I}\right|$ in dotted line and $\left|S_{2 I}\right|$ in solid line. Inset: side-view of the simulated meandered BPF. (b) Photograph of the unassembled prototype.
The in-band return loss will be $25 \mathrm{~dB}$. The slope between the pass- and the stop-band will be reinforced by the introduction of 3 transmission zeros close to the pass-band. The width of the filter is fixed to $a=19.05 \mathrm{~mm}$, and the input port height is $b_{0}=7 \mathrm{~mm}$. Now, using (12), the electrical length results in $\theta_{c}=0.9236 \mathrm{rad}$ for our design. Next, applying the Unit Element extraction procedure, the list of characteristic impedances for the stepped-impedance prototype is obtained: $Z_{0}=Z_{12}=1, Z_{1}=Z_{11}=0.75, Z_{2}=Z_{10}=1.32, Z_{3}=Z_{9}=0.60$, $Z_{4}=Z_{8}=1.62, Z_{5}=Z_{7}=0.53$, and $Z_{6}=1.69$. Then, the required local reflection coefficients $\Gamma_{i}$ are calculated using (2). Now, as explained in Section II.B, each $\pm 90^{\circ} \mathrm{EMB}$ is sequentially computed. For the $i$ th $\pm 90^{\circ} \mathrm{EMB}, d_{i, \text { in }}, d_{i, \text { out }}$, and $b_{i}$ are computed to satisfy (13) (taking into account that $b_{i-1}$ is fixed in the previous $\left.\pm 90^{\circ} \mathrm{EMB}\right)$, while $l_{i, \text { in }}$ and $l_{i, \text { out }}$ are computed to satisfy (14) and (15). Additionally, as explained in Section II.B, each two consecutive $-90^{\circ} \mathrm{EMBs}$ (or $+90^{\circ} \mathrm{EMBs}$ ) can produce a transmission zero, and it will be possible to generate 3 transmission zeros close to the passband in our design. FEST3D is used as electromagnetic simulator for the previous calculations and parameter adjustments. The dimensions obtained are listed in Table III (after applying (16)). The frequency response of the filter designed is plotted in Fig. 14. Although a clear pass-band is achieved, its

\section{TABLE III}

DIMENSIONS OF THE KU-BAND MEANDERED LPF (mm)

\begin{tabular}{ccccccc}
\hline \hline$b_{0}=b_{12}$ & $b_{1}=b_{11}$ & $b_{2}=b_{10}$ & $b_{3}=b_{9}$ & $b_{4}=b_{8}$ & $b_{5}=b_{7}$ & $b_{6}$ \\
\hline 7.000 & 5.775 & 7.965 & 3.561 & 8.951 & 3.224 & 8.843 \\
\hline \hline$l_{0}=l_{12}$ & $l_{1}=l_{11}$ & $l_{2}=l_{10}$ & $l_{3}=l_{9}$ & $l_{4}=l_{8}$ & $l_{5}=l_{7}$ & $l_{6}$ \\
5 & 1.002 & 0.858 & 0.944 & 1.101 & 1.077 & 0.608 \\
\hline \hline$d_{1, \text { in }}=$ & $d_{2, \text { in }}=$ & $d_{3, \text { in }}=$ & $d_{4, \text { in }}=$ & $d_{5, \text { in }}=$ & $d_{6, \text { in }}=$ \\
$d_{12, \text { out }}$ & $d_{11, \text { ut }}$ & $d_{10, \text { out }}$ & $d_{9, \text { out }}$ & $d_{8, \text { out }}$ & $d_{7, \text { out }}$ \\
\hline 0.573 & 3.358 & 2.414 & 1.534 & 1.682 & 2.316 \\
\hline \hline$d_{1, \text { out }}=$ & $d_{2, \text { out }}=$ & $d_{3, \text { out }}=$ & $d_{4, \text { out }}=$ & $d_{5, \text { out }}=$ & $d_{6, \text { out }}=$ \\
$d_{12 \text {,in }}$ & $d_{11, \text { in }}$ & $d_{10, \text { in }}$ & $d_{9, \text { in }}$ & $d_{8, \text { in }}$ & $d_{7, \text { in }}$ \\
\hline 1.275 & 0.558 & 0.365 & 1.919 & 1.601 & 0.960 \\
\hline \hline
\end{tabular}

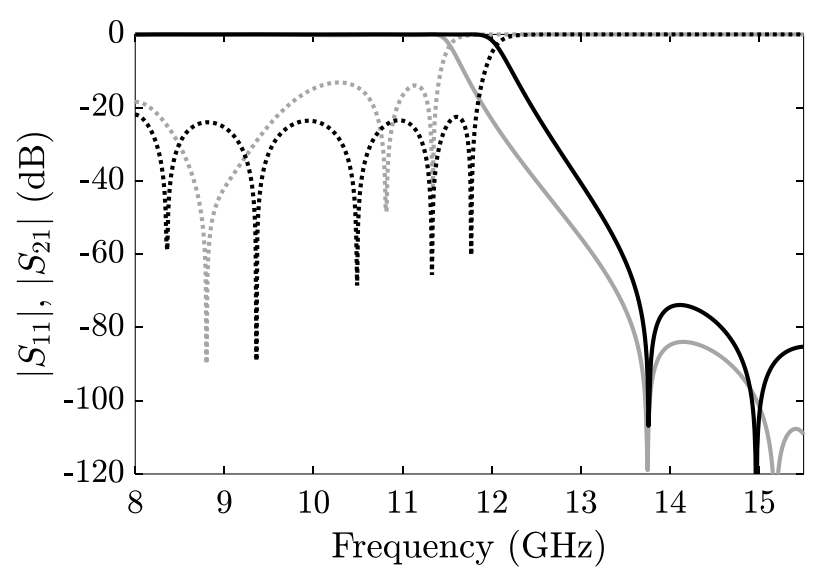

Fig. 14. FEST3D simulated frequency response comparison between the novel LPF designed with the technique in Section II.B (grey line) and after the final adjustment of the filter parameters (black line). $\left|S_{I I}\right|$ in dotted line, $\left|S_{2 l}\right|$ in solid line. 
matching must be improved (obtained return loss better than $15 \mathrm{~dB})$ and a frequency shift appears. To solve those problems, a fine adjustment over the $d_{i}$ 's and $l_{i}$ 's is run. The final dimensions of the filter are detailed in Table IV. The frequency response of the filter obtained after the adjustment fully satisfies the prescribed specifications and is also plotted in Fig. 14. Unlike in the previous meandered BPFs, a final quick optimization over the $d_{i}$ 's and $l_{i}$ 's has been required. This is due to the interaction of the transmission zeros with the implemented Chebyshev frequency response. That interaction cannot be neglected in our case of transmission zeros close to the passband, but it is easily compensated with the final quick optimization over the $d_{i}$ 's and $l_{i}$ 's explained. The novel filter designed in our example has 3 transmission zeros in the required stopband. The first zero is located at $13.75 \mathrm{GHz}$ (produced by the 6 th- 7 th consecutive $-90^{\circ} \mathrm{EMBs}$ ), and a double zero is located at $15 \mathrm{GHz}$ (produced by the 4th-5th consecutive $+90^{\circ} \mathrm{EMBs}$, and their symmetrical 8th-9th consecutive $+90^{\circ} \mathrm{EMBs}$ ). Additionally, 2 transmission zeros (a double zero) located at higher frequencies (not used in our design) are produced by the 2 th- 3 th consecutive $-90^{\circ} \mathrm{EMBs}$,

\section{TABLE IV}

DIMENSIONS OF THE KU-BAND MEANDERED LPF AFTER THE ADJUSTMENT OVER THE $d_{i}$ 's AND $l_{i}$ 's $(\mathrm{mm})$

\begin{tabular}{cccccccc}
\hline \hline$b_{0}=b_{12}$ & $b_{1}=b_{11}$ & $b_{2}=b_{10}$ & $b_{3}=b_{9}$ & $b_{4}=b_{8}$ & $b_{5}=b_{7}$ & $b_{6}$ \\
\hline 7.000 & 5.775 & 7.965 & 3.561 & 8.951 & 3.224 & 8.843 \\
\hline \hline$l_{0}=l_{12}$ & $l_{1}=l_{11}$ & $l_{2}=l_{10}$ & $l_{3}=l_{9}$ & $l_{4}=l_{8}$ & $l_{5}=l_{7}$ & $l_{6}$ \\
5 & 1.002 & 0.858 & 0.05 & 1.101 & 0.05 & 0.608 \\
\hline \hline$d_{1, \text { in }}=$ & $d_{2, \text { in }}=$ & $d_{3, \text { in }}=$ & $d_{4, \text { in }}=$ & $d_{5, \text { in }}=$ & $d_{6, \text { in }}=$ \\
$d_{12, \text { out }}$ & $d_{11, \text { out }}$ & $d_{10, \text { out }}$ & $d_{9, \text { out }}$ & $d_{8, \text { out }}$ & $d_{7, \text { out }}$ \\
\hline 1.000 & 1.163 & 0.810 & 1.676 & 1.652 & 1.949 \\
\hline \hline$d_{1, \text { out }}=$ & $d_{2, \text { out }}=$ & $d_{3, \text { out }}=$ & $d_{4, \text { out }}=$ & $d_{5, \text { out }}=$ & $d_{6, \text { out }}=$ \\
$d_{12, \text { in }}$ & $d_{11, \text { in }}$ & $d_{10, \text { in }}$ & $d_{9, \text { in }}$ & $d_{8, \text { in }}$ & $d_{7, \text { in }}$ \\
\hline 1.001 & 1.498 & 1.231 & 1.997 & 1.564 & 1.068 \\
\hline \hline
\end{tabular}

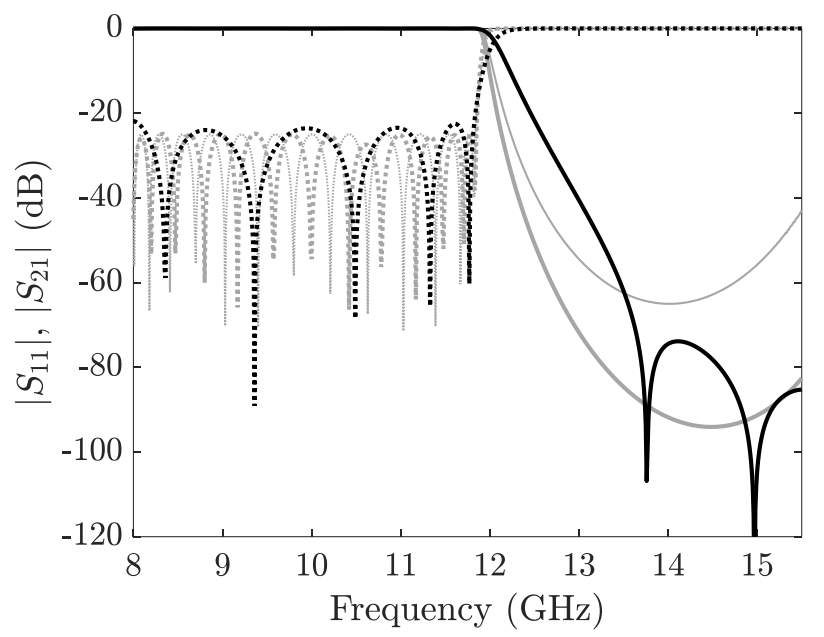

Fig. 15. FEST3D simulated frequency response comparison between the classical straight LPF designed following [32] (grey thin line), the LPF with meandered topology in [33] (grey thick line), and the LPF with transmission zeros designed in this paper (black line), all fulfilling the same frequency specifications. $\left|S_{11}\right|$ in dotted line, $\left|S_{21}\right|$ in solid line. and their symmetrical 10th-11th consecutive $-90^{\circ} \mathrm{EMBs}$.

As a general rule, a filter of order $\mathrm{N}$ (odd for simplicity) will feature $\mathrm{N}+1 \pm 90^{\circ} \mathrm{EMBs}$. Each two consecutive $-90^{\circ} \mathrm{EMBs}$ (or $+90^{\circ} \mathrm{EMBs}$ ) can produce a transmission zero. Assuming that the topology selected is the most compact like in our example (i.e., $+--++--++\cdots--+$ ), there will be (N-1)/2 two-consecutive $-90^{\circ} \mathrm{EMBs}$ (or $+90^{\circ} \mathrm{EMBs}$ ) which can produce a transmission zero. Finally, assuming symmetry in the stepped-impedance prototype (as it happens for Butterworth or Chebyshev functions) the transmission zeros will be grouped in pairs except for a possible single transmission zero.

It is worth noting that the minimum mechanical gap of the final structure obtained in our design example is larger than $3.5 \mathrm{~mm}$, which will provide high-power handling capability as customarily needed. The high-power behaviour of the novel filter has been estimated by means of SPARK3D, using the EM fields previously calculated with CST MWS. The highpower simulation has been performed at $11.7 \mathrm{GHz}$ (upper passband edge, where the EM fields are maximum and the input power threshold will be minimum), using the SEY of silver (ECSS, [36]). The input power theshold level of the proposed LPF is higher than $20 \mathrm{~kW}$, which is a very high value comparable with those that can be found in the literature [23], [37].

The novel meandered LPF with transmission zeros designed has been compared with the classical inline LPF designed following [32], and the meandered LPF structure proposed in [33]. Their simulated frequency responses are compared in Fig. 15, and a side-view of the filters is shown in Fig. 16. As it can be seen, all the filters fulfil the frequency specifications, but the novel filter proposed in this paper is more compact than its counterparts. Actually, the novel filter is only $29.3 \mathrm{~mm}$-long while the filter in [33] is $104.5 \mathrm{~mm}$-long and the inline filter is $180.1 \mathrm{~mm}$-long (more than $70 \%$ and $83 \%$ of length reduction respectively).

The novel meandered LPF with transmission zeros has been fabricated in clam-shell configuration, in bare aluminum, by standard milling with a radius of the drill equal to $1 \mathrm{~mm}$. A photograph of the prototype is shown in Fig. 17. In order to reach the standard WR75 ports for the measurements, two impedance transformers of 1 step have been added to the input

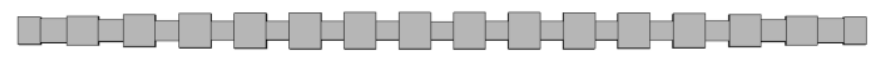

(a)

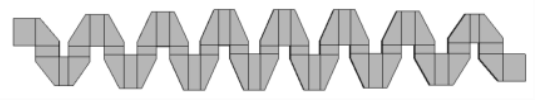

(b)

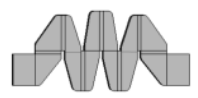

(c)

Fig. 16. Side-view (same scale) of (a) the classical straight LPF designed following [32] (b) the filter with meandered topology in [33], and (c) the filter with transmission zeros designed in this paper, all fulfilling the same frequency specifications. 
and output ports of the structure. The measurement results are also given in Fig. 17. A remarkable agreement between the simulated (including the impedance transformers) and the measured results is obtained. A detail with the insertion loss of the filter at the passband is shown in Fig. 17-inset. As it can be seen, the measured insertion loss for the LPF is below $0.1 \mathrm{~dB}$.

\section{CONCLUSIONS}

In this paper, a new meandered topology for rectangular waveguide LPFs and BPFs is proposed. The novel filters cascade $\pm 90^{\circ} \mathrm{EMBs}$ whose physical dimensions are calculated in a simple modular manner that can be easily integrated in an electromagnetic software tool. Furthermore, due to its modular nature, different lay-out configurations can be achieved without further optimizations, allowing us to obtain ad-hoc designs with routing capabilities to adapt to specific circuit footprints. Additionally, in the case of the novel meandered LPFs, transmission zeros can be introduced, producing very steep slopes between the pass- and the stop-band. Moreover, the novel meandered topology allows for more compact structures in comparison with the state-of-the-art filters. Several examples, three BPFs and one LPF, have been

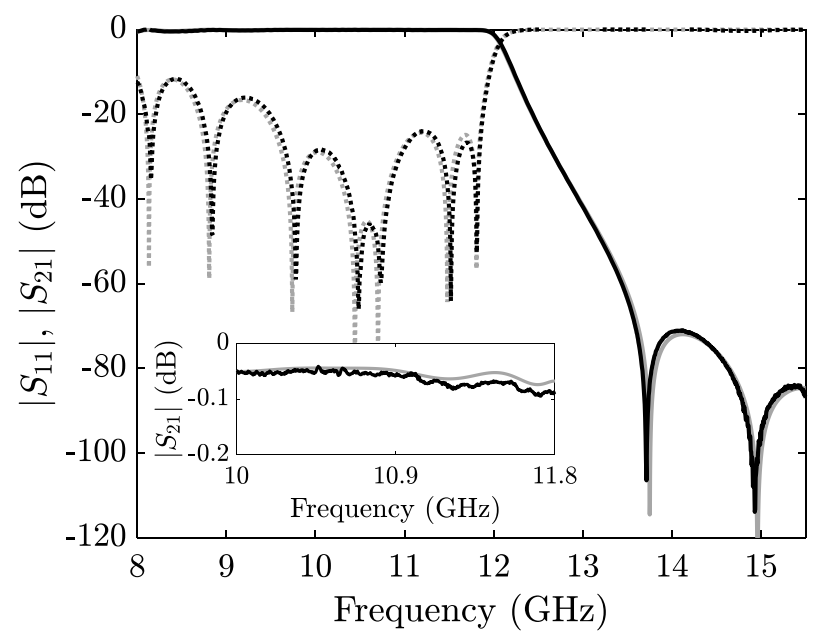

(a)

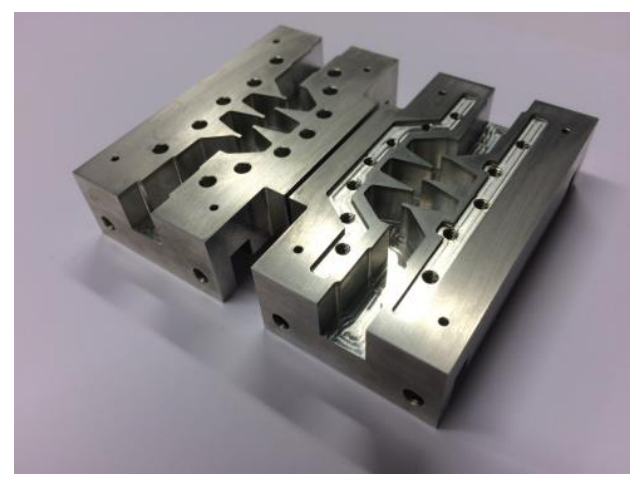

(b)

Fig. 17. Comparison between the CST MWS simulated frequency response (grey line) of the meandered LPF with transmission zeros and measurements of the fabricated prototype (black line). $\left|S_{11}\right|$ in dotted line and $\left|S_{2 l}\right|$ in solid thick line. (b) Photograph of the unassembled fabricated prototype. Inset: Detail of the insertion loss. designed and simulated to demonstrate the aforementioned characteristics. Two BPFs and one LPF have been also fabricated by milling, and the measurements of the prototypes show an excellent agreement with the simulated results, confirming the robustness of the novel filter topology and its design method.

\section{ACKNOWLEDGMENT}

The authors would like to thank the help of Microlan S.L. with the fabrication of the Q-band prototypes.

\section{REFERENCES}

[1] J. Uher, J. Bornemann, and U. Rosenberg, Waveguide Components for Antenna Feed Systems: Theory and CAD. Norwood: Artech House, 1993.

[2] V. Boria and B. Gimeno, "Waveguide filters for satellites," IEEE Microwave Magazine, vol. 8, no. 5, pp. 60-70, Oct. 2007.

[3] R. J. Cameron, C. M. Kudsia, and R. R. Mansour, Microwave Filters for Communication Systems: Fundamentals, Design and Applications. Hoboken, NJ: John Wiley \& Sons, 2007.

[4] M. Skolnik, et al. Radar handbook, Electronics Electrical Engineering, 3rd ed. New York, NY, USA: McGraw-Hill, 2008.

[5] S. Ceccuzzi, C. Ponti, G. L. Ravera and G. Schettini, "Physical mechanisms and design principles in mode filters for oversized rectangular waveguides," in IEEE Transactions on Microwave Theory and Techniques, vol. 65, no. 8, pp. 2726-2733, Aug. 2017.

[6] H. Wolf, M. Schneider, S. Stirland and D. Scouarnec, "Satellite multibeam antennas at airbus defence and space: State of the art and trends," The 8th European Conference on Antennas and Propagation (EuCAP 2014), The Hague, 2014, pp. 182-185.

[7] H. Moheb, C. Robinson and J. Kijesky, "Design \& development of copolarized $\mathrm{Ku}$-band ground terminal system for very small aperture terminal (VSAT) application," IEEE Antennas and Propagation Society International Symposium. 1999 Digest. Held in conjunction with: USNC/URSI National Radio Science Meeting (Cat. No.99CH37010), Orlando, FL, USA, 1999, pp. 2158-2161 vol.3.

[8] X. Zhao et al., "Analytic passive intermodulation model for flange connection based on metallic contact nonlinearity approximation," in IEEE Transactions on Microwave Theory and Techniques, vol. 65, no. 7, pp. 2279-2287, July 2017.

[9] C. Vicente, D. Wolk, H. L. Hartnagel and D. Raboso, "An Experimental Investigation on Passive Intermodulation at Rectangular Waveguide Interfaces," 2006 IEEE MTT-S International Microwave Symposium Digest, San Francisco, CA, 2006, pp. 242-245.

[10] A. Morini, T. Rozzi and M. Mongiardo, "Curved filters in rectangular waveguide," 1996 26th European Microwave Conference, Prague, Czech Republic, 1996, pp. 153-154.

[11] Chi Wang, K. A. Zaki, A. E. Atia and T. G. Dolan, "Dielectric combline resonators and filters," in IEEE Transactions on Microwave Theory and Techniques, vol. 46, no. 12, pp. 2501-2506, Dec 1998.

[12] Ming Yu, Wai-Cheung Tang, A. Malarky, V. Dokas, R. Cameron and Ying Wang, "Predistortion technique for cross-coupled filters and its application to satellite communication systems," in IEEE Transactions on Microwave Theory and Techniques, vol. 51, no. 12, pp. 2505-2515, Dec. 2003.

[13] T. Shen and K. A. Zaki, "Folded evanescent-mode ridge waveguide bandpass filters," 2001 31st European Microwave Conference, London, England, 2001, pp. 1-4.

[14] E. Ofli and R. Vahldieck, "A novel compact millimeter wave diplexer," 2002 IEEE MTT-S International Microwave Symposium Digest (Cat. No.02CH37278), Seattle, WA, USA, 2002, pp. 377-380 vol.1.

[15] M. Guglielmi, F. Montauti, L. Pellegrini and P. Arcioni, "Implementing transmission zeros in inductive-window bandpass filters," in IEEE Transactions on Microwave Theory and Techniques, vol. 43, no. 8, pp. 1911-1915, Aug 1995.

[16] C. Tomassoni, L. Marcaccioli, M. Dionigi, M. Mongiardo, R. V. Gatti and R. Sorrentino, "CAD of folded filters and diplexers by the generalized scattering matrix of the single step discontinuity," 2004 IEEE MTT-S International Microwave Symposium Digest (IEEE Cat. No.04CH37535), 2004, pp. 1843-1846 Vol.3. 
[17] J. Bornemann, S. Amari and R. Vahldieck, "A flexible S-matrix algorithm for the design of folded waveguide filters," 2005 European Microwave Conference, 2005, pp. 4.

[18] C. Carceller, P. Soto, V. Boria, M. Guglielmi and D. Raboso, "New folded configuration of rectangular waveguide filters with asymmetrical transmission zeros," 2014 44th European Microwave Conference, Rome, 2014, pp. 183-186.

[19] U. Rosenberg, M. Knipp and S. Amari, "Compact diplexer design using different E-plane triplets to serve contiguous passbands with high interband selectivity," 2006 European Microwave Conference, Manchester, 2006, pp. 133-136.

[20] C. Carceller, P. Soto, V. E. Boria and M. Guglielmi, "Design of hybrid folded rectangular waveguide filters with transmission zeros below the passband," IEEE Transactions on Microwave Theory and Techniques, vol. 64, no. 2, pp. 475-485, Feb. 2016.

[21] F. Teberio et al., "Chirping techniques to maximize the power-handling capability of harmonic waveguide low-Pass filters," in IEEE Transactions on Microwave Theory and Techniques, vol. 64, no. 9, pp. 2814-2823, Sept. 2016.

[22] I. Arregui et al., "High-power low-pass harmonic filters with higherorder TEn0 and non-TEn0 mode suppression: design method and multipactor characterization," in IEEE Transactions on Microwave Theory and Techniques, vol. 61, no. 12, pp. 4376-4386, Dec. 2013.

[23] O. A. Peverini et al., "Enhanced topology of E-plane resonators for high-power satellite applications," in IEEE Transactions on Microwave Theory and Techniques, vol. 63, no. 10, pp. 3361-3373, Oct. 2015.

[24] Leo Young, "Stepped-impedance transformers and filter prototypes," in IRE Transactions on Microwave Theory and Techniques, vol. 10, no. 5, pp. 339-359, September 1962.

[25] G. L. Matthaei, L. Young, and E. M. T. Jones, Microwave Filters, Impedance-Matching Networks, and Coupling Structures. Norwood: Artech House, 1980.

[26] S. B. Cohn, "A theoretical and experimental study of a waveguide filter structure," Office Naval Res., Cruft Lab., Harvard Univ., Cambridge, Mass., Rep. 39, Apr. 25, 1948.

[27] R. Levy, "Tables of element values for the distributed low-pass prototype filter," IEEE Transactions on Microwave Theory and Techniques, vol. 13, no. 5, pp. 514-536, Sep 1965.
[28] R. Levy, "A generalized design technique for practical distributed reciprocal ladder networks, " IEEE Transactions on Microwave Theory and Techniques, vol. 21, no. 8, pp. 518-526, Aug. 1973.

[29] R. Levy, "Tapered corrugated waveguide low-pass filters," IEEE Transactions on Microwave Theory and Techniques, vol. 21, no. 8, pp. 526-532, 1973.

[30] M. Simeoni, S. Cacchione, F. Vanin, J. Molina-Perez, and D. Schmitt, "Automatic dimensional synthesis without optimization for stepped impedance low-pass filters," Microwave Optical and Technology Letters, vol. 44, no. 2, pp. 190-194, 2005.

[31] V. E. Boria, P. Soto and S. Cogollos, "Distributed models for filter synthesis," IEEE Microwave Magazine, vol. 12, no. 6, pp. 87-100, Oct. 2011.

[32] F. Teberio, I. Arnedo, J. M. Percaz, I. Arregui, T. Lopetegi and M. A. G. Laso, "Accurate design of corrugated waveguide low-pass filters using exclusively closed-form expressions," 2017 47th European Microwave Conference (EuMC), Nuremberg, 2017, pp. 632-635.

[33] F. Teberio et al., "Meandered corrugated waveguide low-pass filter," 2017 IEEE MTT-S International Microwave Workshop Series on Advanced Materials and Processes for $\mathrm{RF}$ and $\mathrm{THz}$ Applications (IMWS-AMP), Pavia, 2017, pp. 1-3.

[34] R. Levy, "Inhomogeneous stepped-impedance corrugated waveguide low pass filters," IEEE MTT-S International Microwave Symposium Digest, 2005., 2005, pp. 4.

[35] F. Teberio et al., "Waveguide band-pass filter with reduced sensitivity to fabrication tolerances for Q-band payloads," 2017 IEEE MTT-S International Microwave Symposium (IMS), Honololu, HI, 2017, pp. 1464-1467.

[36] ESA-ESTEC, Space Engineering: Multipacting, Design and Test, vol. ECSS-20-01A, edited by ESA Publication Division, the Netherlands.

[37] P. Sarasa, A. Gonzalez, H. Esteban, P. Mader, K. Tossou, P. Lepeltier, "Comparative study of the power handling capability of space broadband antenna filters in ku-band," in Proc. $5^{\text {th }}$ International Workshop on Multipactor, Corona and Passive Intermodulation in Space RF Hardware (MULCOPIM 2005), pp. 93-99, ESA-ESTEC, Noordwijk, The Netherlands, 2005. 